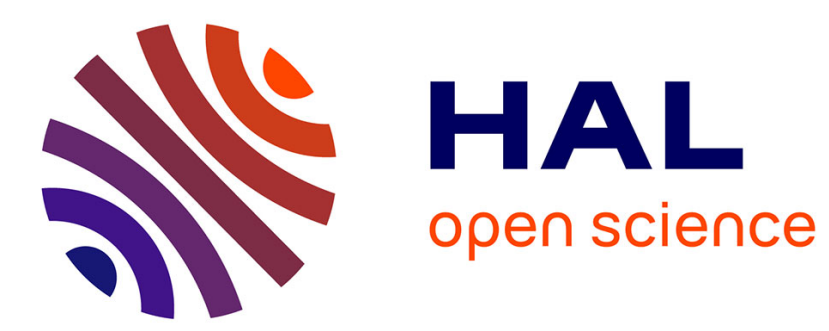

\title{
TREATMENT OF HEAVY ION COLLISIONS AT INTERMEDIATE ENERGIES
}

\author{
Joerg Aichelin
}

\section{To cite this version:}

Joerg Aichelin. TREATMENT OF HEAVY ION COLLISIONS AT INTERMEDIATE ENERGIES. Journal de Physique Colloques, 1986, 47 (C4), pp.C4-63-C4-75. 10.1051/jphyscol:1986406 . jpa00225769

\section{HAL Id: jpa-00225769 https://hal.science/jpa-00225769}

Submitted on 1 Jan 1986

HAL is a multi-disciplinary open access archive for the deposit and dissemination of scientific research documents, whether they are published or not. The documents may come from teaching and research institutions in France or abroad, or from public or private research centers.
L'archive ouverte pluridisciplinaire HAL, est destinée au dépôt et à la diffusion de documents scientifiques de niveau recherche, publiés ou non, émanant des établissements d'enseignement et de recherche français ou étrangers, des laboratoires publics ou privés. 
JOURNAL DE PHYSIQUE

Colloque $C 4$, supplément au $\mathrm{n}^{\circ} 8$, Tome 47 , août 1986

C4-63

TREATMENT OF HEAVY ION COLLISIONS AT INTERMEDIATE ENERGIES

\section{J. AICHELIN}

Institut für Theoretische Physik der Universität Heidelberg and

Max-PIanck-Institut für Kernphysik, D-6900 Heidelberg, F.R.G.

Abstract - Microscopic calculations of intermediate energy heavy ion collision are performed employing the Boltzmann Uehling Uhlenbeck (BUU) formalism. We see that at beam energies around the Fermi energy the system does not equilibrize although the spectra show an exponential form. Preeguilibrium particles are preferably emitted to the opposite site of the impact parameter as suggested by the Fermi jet model. We discuss furthermore the linear momentum transfer, the nuclear mean free path and the formation of a third cluster at midrapidity which reflects nicely the importance of both - two body collisions and mean field - at these beam energies.

\section{I - INTRODUCTION}

At low as well as at high beam energies (Ekin/ $<10 \mathrm{MeV}$ and $>200 \mathrm{MeV}$ ) heavy ion reactions can be understood in simple models. At low beam energy with increasing impact parameters we see the formation of compound nuclei, deep inelastic processes and peripheral reactions. At high beam energies the single particle inclusive data can be well described assuming that the geometrically overlapping zones form an equilibrated source of particle emission. So at low and at high beam energies we observe equilibration, however, the mechanism which drives the system towards equilibrium is completely different.

At low energies only very few particles can escape from the combined system of projectile and target. The attractive mean field binds the nucleons together long enough to equilibrize although the residual twobody interaction is highly suppressed due to the Pauli principle which hinders the scattering in already occupied states. At high energies the mean field plays no role anymore being negligible compared to the average kinetic energy of the nucleons. At these energies however the Pauli principle is less severe and consequently the mean free path for nucleon nucleon collisions decreases. Therefore mutual two-body interactions which occur while projectile and target move through each other are sufficient to equilibrize the interaction zone.

When a while ago heavy ion beams between Ekin $=10 \mathrm{MeV} / \mathrm{N}$ and $200 \mathrm{MeV} / \mathrm{N}$ became available first experiments indicated [1] that single particle inclusive spectra also show the exponential forms which one would expect for particle emission from an equilibrated source. However the value of the slope parameter cannot easily be reconciled with the expected temperature of the total system or a geometrically defined subsystem. So it remained a question to theory whether also at intermediate energies a thermal system can be identified. 
Another topic of current interest is the question which mechanism Ieads to the multifragmentation of the target into several fragments of charges larger than 2. This process has first been established at high beam energies but even at energies as $10 \mathrm{w}$ as $84 \mathrm{MeV} / \mathrm{N}$ [2] we see the same mechanism at work. At even lower energies the situation is more puzzling. Multifragmentation occurs in central collisions and is therefore associated with a large multiplicity of nucleons. Although it seems that multifragmentation is still observed at $30 \mathrm{MeV} / \mathrm{N}[3]$, at this energy also coincidences between projectile like fragments and light clusters are reported [4]. A theoretical explanation of the underlying mechanism of this kind of peripheral reactions is not at hand.

At energies below $10 \mathrm{MeV} / \mathrm{N}$ the linear momentum transfer between projectile and target is complete. At higher energies less and less momentum can obviously be transferxed [5]. Is this due to peripheral reactions in which the projectile is cleaved and only part of it transfer momentum whereas the rest moved almost uninfluenced, or is it due to the fact that the nuclear stopping power is limited and therefore even in central collisions the projectile cannot completely be stopped at this energy?

Already at moderate beam energies the single particle inclusive data can be described as a sum of two components, both exponential in form but with different slope parameters [6]. The low energy component can be well understood assuming that the particles stem from compound decay. The hard component was phenomenologically described as resulting from a Fermi jet mechanism which emerges from the interaction region prior to equilibration [7].

All these points intensified the demands for a comprehensive theory of heavy ion reactions which includes both, mean field dynamics which governs nuclear reactions at low energies as well as two-body collisions which dominates at high energies. The first results of such a theory are obtained by now. I will concentrate here on the above mentioned questions : a) Is the Fermi Jet mechanism the true source of preequilibrium particle emissions at energies around $25 \mathrm{MeV} / \mathrm{N}$ ? b) why are three large clusters formed at beam energies around the Fermi energy? c) Does thermalization occur in medium energy heavy ion reaction? d) How much momentum can be transferred in this reaction and does there exist an upper limit as indicated by experiments?

\section{II - BUU THEORY.}

In principal one would like to know the time evolution of the $A_{t}+A_{p}$ body density matrix which is formally expressed by the von Neumann equation. However neither do we know the initial $n$ body density matrix nor do we know how to solve this equation. Furthermore we are not interested in all informations contained in this equation but rather in that part which dominates the time evolution.

Rather than to introduce approximations into the quantum mechanical von Neumann equation we choose a different ansatz. We start with the classical analogon - the Liouville equation - and put in the dominating quantum mechanical features by hand. It will be shown later that on the one-body level, the time evolution of the classical phase space density and its quantummechanical counterpart is almost identical.

In our calculations $[8,9]$ the nucleons are classical points like particles in coordinate and momentum space. Initially we assign to each nucleon a position in a sphere of $\mathrm{R}=1.2 \mathrm{a} * * 1 / 3$. and a momentum between $o$ and $p_{f}$ randomiy. The Fermi momentum $p_{f}$ is determined by a local Fermi gas approximation. This procedure guarantees that on the average each nucleon occupies initially a phase space volume of $h * * 3 / 4$. The nucleons interact via stochastic two-body scattering with an isotropic cross 
section of $40 \mathrm{mb}$ as well as via a selfconsistent density dependent mean potential :

$$
\mathrm{U}(\rho)=\mathrm{a} *_{\rho}+\mathrm{b} *_{\rho} * *_{\mathrm{C}}
$$

For the calculations presented here we use $a=-353 \mathrm{MeV}, \mathrm{b}=303 \mathrm{MeV}, \mathrm{c}$ $=7 / 6$ which gives a compressibility of $200 \mathrm{MeV}$. When two nucleons come closer than $\mathrm{d}=J(\sigma / \pi)$ the nucleons may scatter. We check whether the scattering violates the Pauli principle by examining the phase space around the phase space coordinates of the scattered particles. We calculate that part of this phase space volume $(\Omega)$ which is already occupied by other particles $(\Delta \Omega)$. Finally. We determine the scattering probability by $p=\max [1-\Delta \Omega / \Omega, 0]$. The total scattering probability is the product of that of both collision partners.

On the one-body level the equation we solve is identical with the Boltzmann equation with an Uehling Uhlenbeck scattering term. In contrary to the Boltzmann scattering term this scattering term takes care of the Pauli blocking and can be considered as a first order approximation to an exact quantal scattering term. In its underlying equation, however not in the numerical methods applied, it is identical with the so called vUU calculations [9].

Since our approach is not a straightforward derivation of the underlying quantum mechanical $n$ body equation we have first of all to make sure that that it contains the essential ingredients for the time evolution of the system of interest. The best method to prove this is a comparison between the classical mean field calculation and its quantal equivalent, the Time Dependent Hartree Fock calculation [10]. This comparison is shown in figure 1 for the system $84 \mathrm{MeV} / \mathrm{N} C+\mathrm{C}$ at $\mathrm{b}=\mathrm{I} \mathrm{fm}$. Column 1 and 2 show the density at different times for the quantal and the classical mean field calculation. We see an almost complete agreement of the quantities of interest, the linear momentum transfer and the deflection angle. We can therefore conclude that at these energies the detailed form of the wavefunction is irrelevant for the time evolution of the system. Choosing the coordinates of the nucleons randomly, as done in the classical calculation, yields the same result as determining the slater determinants properly by solving the schroedinger equation. In column 3 we see the time evolution of the system if we allow Pauli corrected collision. We observe a completely different time evolution : Besides remnants we see a midrapidity source of particle emission. These nucleons have suffered at least one collision. This midrapidity source is essential to describe the relatively mild angular dependence of the inclusive proton spectrum in the nucleus nucleus center of mass system $[1]$. 


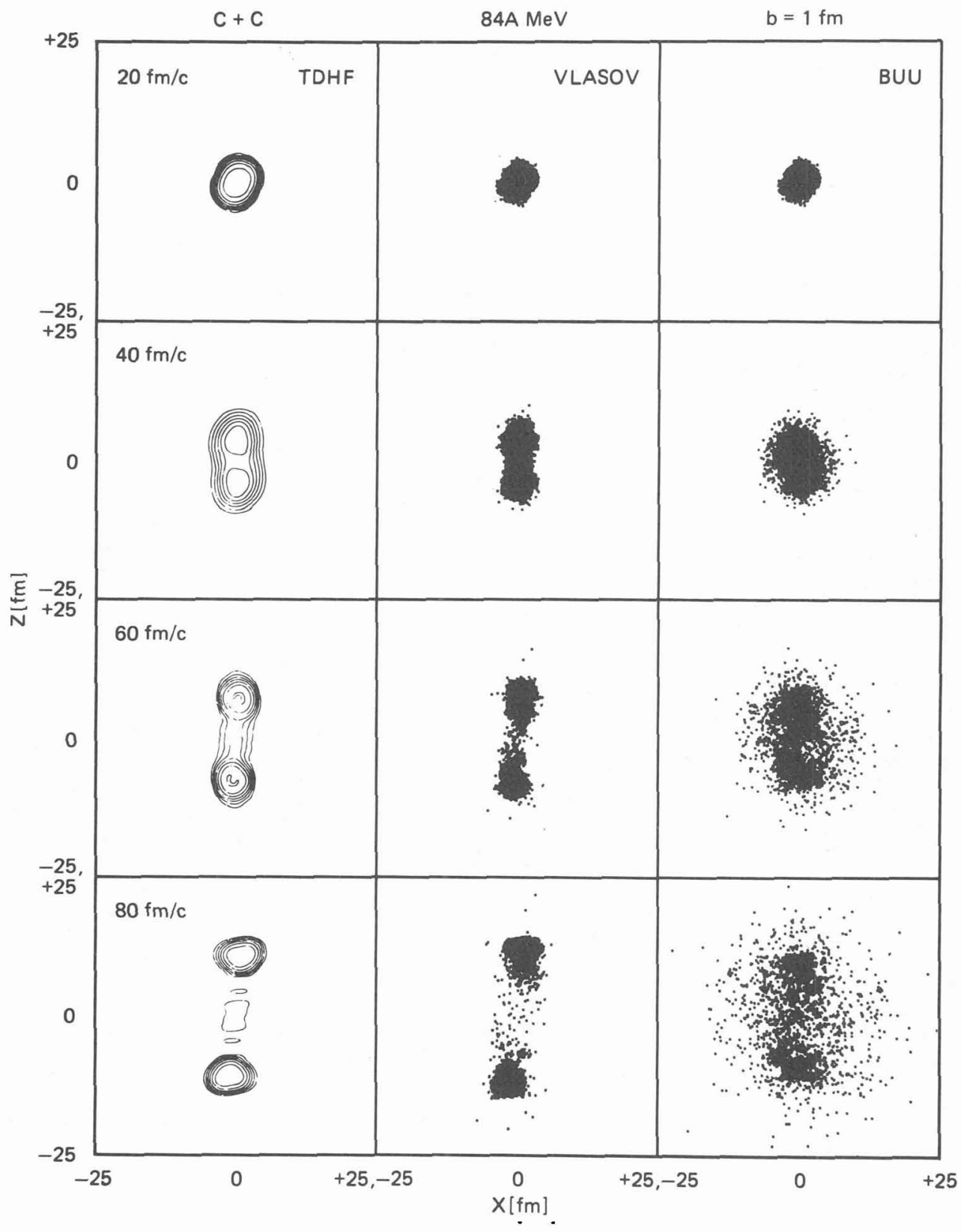

Fig. 1 : Time evolution of the single particle distribution function for the reaction $C(85 \mathrm{MeV} / \mathrm{N})+C$ at $b=1 \mathrm{fm}$ as predicted by the quantal mean field theory without collision term (TDHF, left-hand side column), the classical mean field theory (Vlasov's equation without collision term, center column) and the Vlasov equation with a Uehling-Uhlenbeck collision term included (right-hand side column). The transparency is evident in both mean field results without collision term. In contrast, a rapid deceleration of $60 \%$ of the incident nuclei is observed once the collision term is included into the vlasov equation. 


\section{III - PREEOUILIBRIUM EMISSION OF FAST PARTICLES - FERMIJETS}

$25 \mathrm{AMeV} \mathrm{C}+\mathrm{Au}$
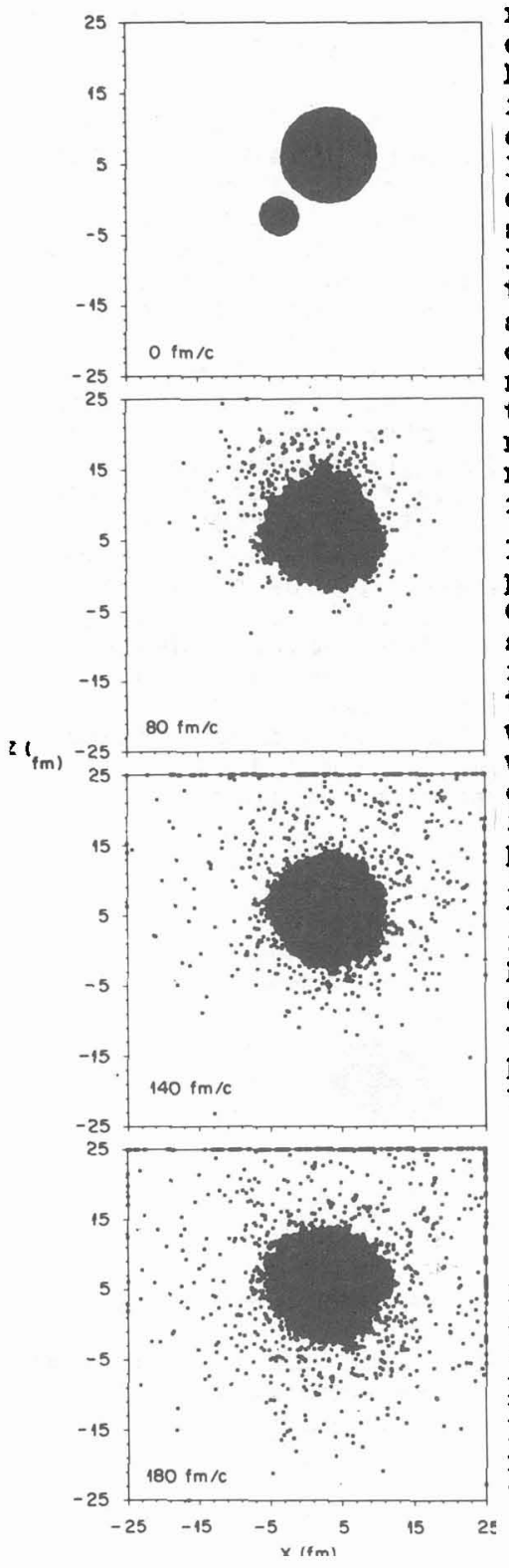

The spectra of protons emitted in heavy ion reactions for incident energies ranging from a few MeV up to $20 \mathrm{MeV} / \mathrm{n}$ above the coulomb barrier cannot be completely explained in terms of emission from a thermalized system [6]. At high energies and in forward direction there is a considerable enhancement indicating an emission prior to equilibrium. This nonequilibrium component has been phenomenologically explained by a jet mechanism [7]. It is assumed that projectile nucleons from the overlap region of projectile and target can travel through the target without suffering collisions and are only little affected by the nuclear mean field. For some of these projectile nucleons the relative momentum with respect to the target surface is enhanced by the Fermi motion. Those are assumed to be able to surmount the potential barrier at the target surface and escape.

In order to verify this reaction mechanism we performed calculation for the system $25 \mathrm{MeV} / \mathrm{N}$ $C+A u$ at $b=7 \mathrm{fm}$. The time evolution of this system is displayed in figure 2. For four different $t$ imes $(t=0,80,140,180 \mathrm{fm} / \mathrm{c})$ we see the projected density distribution. At $80 \mathrm{fm} / \mathrm{c}$ we see the emission of many particles in forward direction in the nucleus nucleus center of mass system whereas only very few are seen in the backward direction. There is no large projectile remnant. The attractive mean field is sufficiently strong to absorb the projectile although initialiy there was less than $50 \%$ overlap. At $140 \mathrm{fm} / \mathrm{c}$ we see a strong enhancement of particle emission opposite to the direction of the impact parameter. Only in the very late stage of the reaction the emission pattern is fairly isotropic as expected from the decay of a compound nucleus.

Fig. 2 : survey of the reaction 25A MeV C+Au at $b=7 \mathrm{fm}$. Here the coordinates of all particles of 100 simulations of the reaction are projected onto the $\mathrm{xz}$ plane, were $\mathrm{z}$ is the beam direction and $x$ the direction of the impact parameter. The density distribution is plotted at $t=0,80,140,180 \mathrm{fm} / \mathrm{c}$. 
The left-right asymmetry of the emission get even more pronounced tagging the particles according their kinetic energy in the nucleus nucleus center of mass system. Particles with Ekin $<25 \mathrm{MeV}$ show an almost isotropic distribution : 3.07 (3.53) have negative (positive) $p_{x}$ values. The energetic particles, however, are most probably emitted on the opposite site to the impact parameter. We see 1.59 (1.13) particles with $p_{x}>0\left(p_{x}<0\right)$. This findings were recently confirmed by experiment [il].

Where were these energetic particles located at the beginning of the reaction? since our theory deals with classical particles we can trace back the momentum and position of the particles in time. In figure $3 a$ we display the position at $t$ ime $t=20 \mathrm{fm} / \mathrm{c}$ of those particles which were finally emitted with energies laxger than $25 \mathrm{MeV}$, in figure $3 \mathrm{~b}$ we display the same quantity for all enitted particles. We see that the energetic particles come with a high probability from the overlap region. The targetlike nucleons are predominantly scatiering partners of projectile nucleons. They have exchanged the momentum with the projectile nucleons due to the isotropic cross sections. The projectile nucleons of figure $3 \mathrm{a}$ have also a $30 \%$ higher momentum compared to the average of all projectile nucleons. As already suggested in the phenomenological models, additional momentum from the Fermi motion helps to overcome the potential barrier at the surface of the target. However, there is one mechanism present which is not counted for in these models : The absorption of the projectile generates a zone of higher density. The mean field tries to lower the density in this zone by accelerating particles in the direction of low density regions. Hence also the energetic projectile nucleons get an extra push which makes them even faster compared to the velocity of the target, thus enhancing their probability of escaping from the system. The lower energy particles are much more isotropically alstributed over projectile and target as may be seen from figure $3 \mathrm{~b}$. They reflect almost an emission from an equilibrated system.
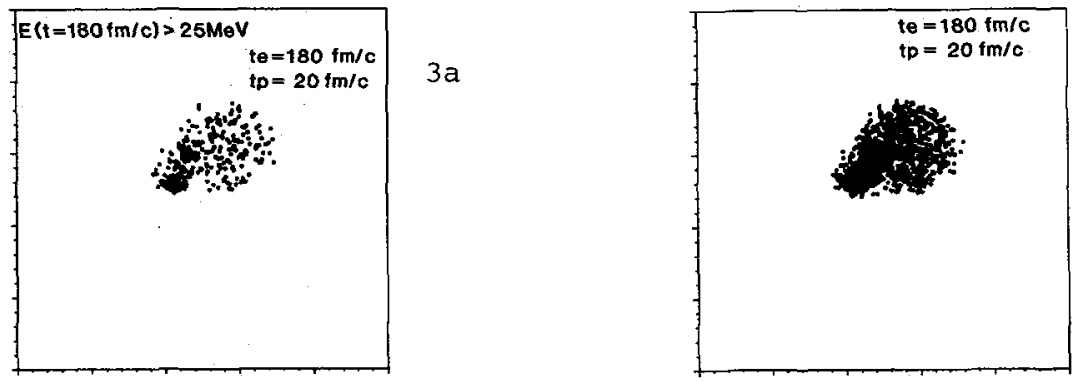

Fig. 3 : Density profile of the emitted particles. The position at time $t p$ is displayed of all those particles which are emitted at time te. Fig. $3 a$ shows those particles whose einal Ekin is larger than $25 \mathrm{MeV}$. Fig. 3b displays all emitted particles.

\section{IV - FORMATION OF THREE HEAVY CLUSTERS}

The formation of three large cluster is another phenomenon which is predicted by the BUU theory for energies around the Fermi energy. It is formed by a complicated interplay between two-body collisions and the nuclear mean field. Figure 4 displays the density profile for the reaction $44 \mathrm{MeV} / \mathrm{N}$ Art $\mathrm{Ni}$ for four different impact parameters. The coordinates of all particles of 100 simulations are projected onto the $\mathbf{x z}$ 


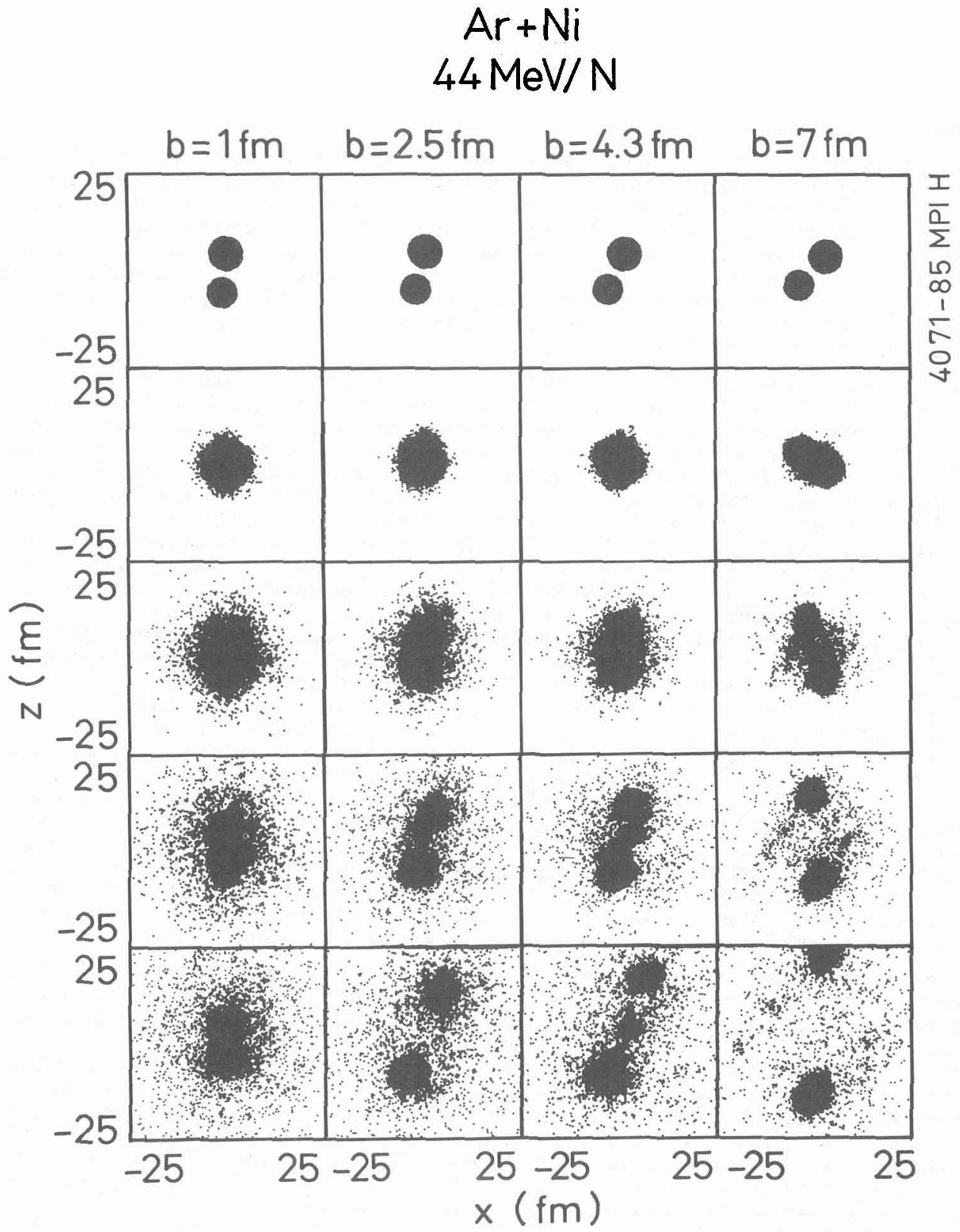

Fig. 4 : Survey of the reaction $44 \mathrm{~A} \mathrm{MeV} \mathrm{Ar+Ni} \mathrm{at} \mathrm{different} \mathrm{impact}$ parameters. Here the coordinates of all particles of 100 simulations of the reaction are projected onto the $x z$ plane, where $z$ is the beam direction and $x$ the direction of the impact parameter. The density distribution is plotted at $t=0,40,80,120,180 \mathrm{fm} / \mathrm{c}$. 
plane where $z$ is the beam direction and $x$ is the direction of the impact parameter. The density profile is plotted at $t=0,40,80,120,180$ $\mathrm{fm} / \mathrm{c}$ from top to bottom. At low impact parameters the system fuses. At $\mathrm{b}=4.3 \mathrm{fm}$ we see at $t=180 \mathrm{fm} / \mathrm{c}$ three large stable clusters which are well separated from each other. At larger and smaller impact parameters we see finally two clusters only.

This is first of all a completely unexpected phenomenon considering the condition under which three clusters may be observed in BUU calculations. The BUU calculation is essentially a mean field calculation in which all fluctuations are strongly damped. If multifragmentation is induced by density fluctuations we would not expect to see it in a BUU calculation. Here only reaction mechanism survive which are very close to the average behaviour of the time evolution of the system. observing three clusters means therefore that this is the dominant process and not caused by fluctuations.

What is the driving mechanism behind this process ? This can be inferred from figure 5 where the position of the center of mass of different types of nucleons are plotted as a function of time. Depending where the nucleons were at $t=0$ and $t=180 \mathrm{fm} / c$, we distinguish six classes i.e all possible combinations of origins (target, projectile), and final destinations ( target remnant, projectile remnant and midrapidity cluster). The thickness of the lines is proportional to the number of class members. For $b=2.5 \mathrm{fm}$ we see only four classes because there exists no third cluster as can be seen from figure 4. Here some of the nucleons from the geometrical overlap move from projectile to target or vice versa and get dragged along with the host nucleus. At $b=4.3$ fm this classes of nucleons exist as well, but in addition a third cluster is formed. Initially these nucleons are placed between the both aforementioned classes. They get less accelerated by the mean field - which wants to lower the density in the overlap region - than those finaliy absorbed by the host nucleus, but much more than those which keep their identity as target or projectile nucleons. Because the overlap is smaller in size, there are less collisions, the total linear momentum transfer between projectile and target is smaller and consequently projectile and target remnant separate much faster at $b=4.3 \mathrm{fm}$ compared to $\mathrm{b}=2.5 \mathrm{fm}$. So at $\mathrm{b}=2.5 \mathrm{fm}$ the remnants have still the possibility to reabsorb those nucleons which potentially would form the third cluster. At $b=4.3 \mathrm{fm}$ the remnants have lost this ability. Rather the system disrupts at the surface of the remnants, forming a cluster almost at rest in the nucleus nucleus center of mass.

Summarizing these observations we see in the reaction $44 \mathrm{~A} \mathrm{MeV} \mathrm{Ar+Ni}$ a third heavy cluster for a range of intermediate impact parameters. It is created by a cooperation of the mean field and collisions. Collisions cause a rapid deceleration which is necessary to initialize the formation of the third clusters. Therefore this process is not seen in time dependent Hartree Fock (TDHF) calculations. The mean field however is responsible for the acceleration of those nucleons in transverse direction which directs the nucleons in different regions in space. A neck is formed. At low impact parameters resp. larger linear momentum transfer the potential field of the remnants is strong enough to accelerate the neck nucleons considerably towards the center of one of the remnants. So the neck nucleons can catch up and get absorbed. The systems disrupts in the midale of the neck. For smaller momentum transfer resp. larger impact parameters, projectile and target remnants emerge faster from the interaction region and consequently their ability to absorb the neck nucleons get reduced. Furthermore the neck nucleons have a larger transverse momentum which enlarge the momentum transfer necessary to catch up with the remnants. Both effects together hinder an absorption of the neck nucleons. The system disrupts at the surface of the remnants. The interacting region contains many nucleons at reasonable high density. It can therefore stabilize and forms a third cluster. 

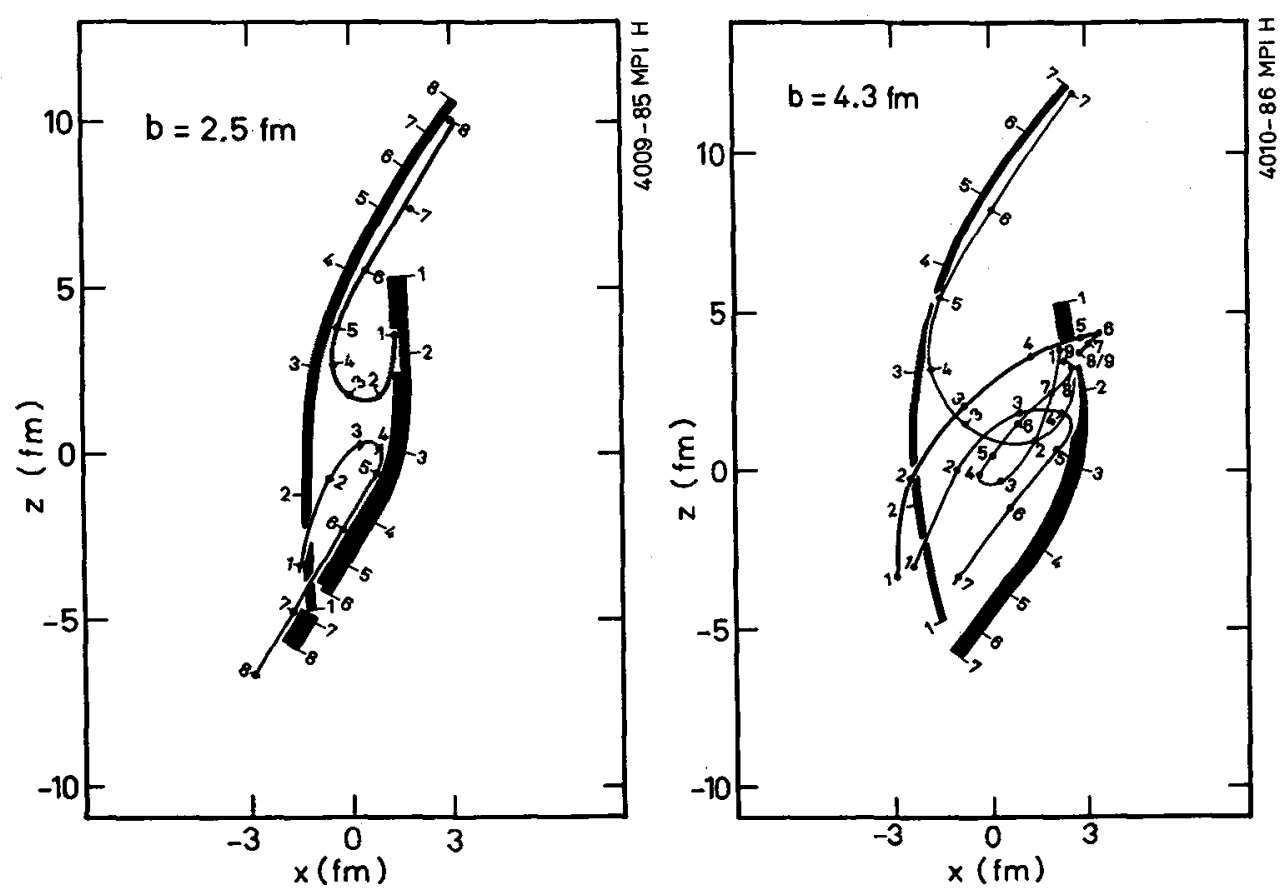

Fig. 5 : Mean positions of different classes of nucleons for two impact parameters $(b=2.5 \mathrm{fm}$ and $b=4.3 \mathrm{fm})$. We see the mean values of those nucleons which belong finally to projectile remnant, target remnant or to the third cluster. The thickness of the lines is proportional to the number of nucleons which belong to each class. The numbers mark the center of mass of each class in timesteps of $20 \mathrm{fm} / \mathrm{c}$.

However, even at the same energy per nucleon we do not see a third cluster in case of a carbon-projectile. For the formation of the third cluster we need two different classes of nucleons : those which are only little decelerated and those whose longitudinal momentum is almost zero in the nucleus nucleus center of mass frame but have gained transverse momentum. Only if both classes can be formed simultaneously the system disrupts at the surface of projectile and target remnant. Small projectiles are insufficient for this process for two reasons. First of all they cannot simultaneously provide enough nucleons for a remnant, large enough to be a stable cluster, and for a dens neck region, large enough to stabilize. In addition also the viscosity is too large. In small projectiles the possible velocity difference between the leftmost and rightmost nucleons is limited. The outermost nucleons are influenced if the innermost nucleons get decelerated whereas here (at $t=$ $40 \mathrm{fm} / \mathrm{c}$ and $b=4.3 \mathrm{fm}$ ) the most left resp. right nucleons move with projectile resp. target velocity with an almost linear velocity profile in between.

\section{$V$ - THERMALIZATION AT INTERMEDIATE ENERGIES.}

At low (Ekin $<5 \mathrm{MeV} / \mathrm{N}$ ) as well as at very high energies (Ekin > 200 $\mathrm{MeV} / \mathrm{N}$ ) the inclusive single particle energy spectra are exponential in shape and the angular distribution is almost isotropic in the rest system of the compound system resp. the fireball. This has been inter- 
preted in terms of an emission from an equilibrated system. Figure 6 shows the proton spectra in the reaction $84 \mathrm{MeV} / \mathrm{N} \mathrm{C}+\mathrm{Au}$ comparing the theory with results of Jakobsson et al [1]. We see that also at intermediate energies the high energy part of the spectra looks quite exponential. However, the slope parameter is larger than expected from a

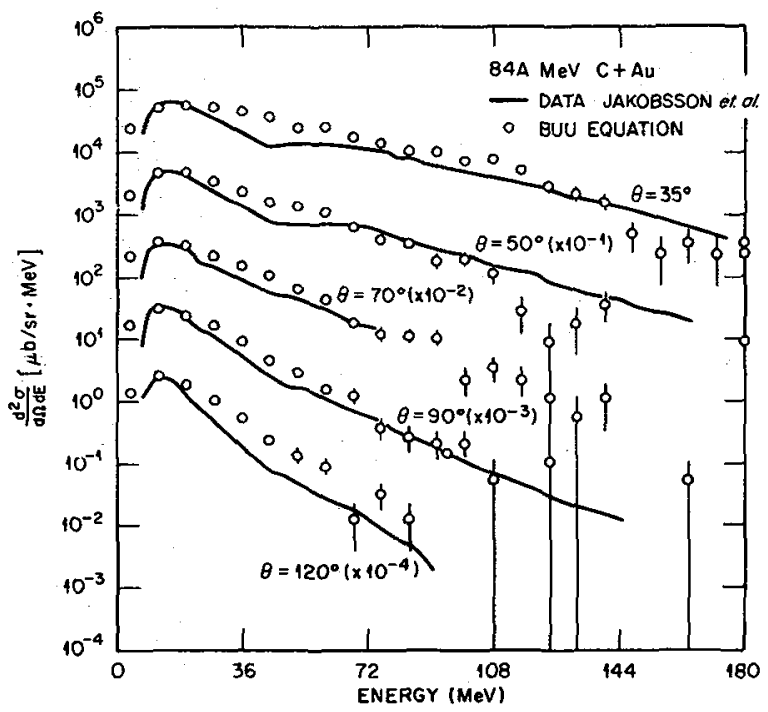

Fig. 6 : The proton spectrum in the reaction $84 \mathrm{Mev} / \mathrm{N} \mathrm{C}+\mathrm{Au}$ comparing the theory with results of Jakobsson et al.[1]. The curves show the energy spectra at angles of $35^{\circ}, 50^{\circ}, 65^{\circ}, 110^{\circ}, 135^{\circ}$ with curves displaced by a factor of 10 for clarity in the figure.

compound nucleus and smaller than expected from a fireball model. In order to connect this exponential form of the spectrum with a temperature we have to find the emitting source. Jakobsson has shown that the spectrum can be well described using three sources with different temperatures and different velocities. In order to check whether the situation is that simple and lacks any sign of preequilibrium we calculate the first and second moment of the momentum distribution of the emitted particles as a function of their emission time: These quantities are displayed in figure 7 . We see that the average momentum of the emitted particles with respect to the nucleus nucleus center of mass system as well as the width of the momentum distribution are strongly dependent on the emission time. At the beginning of the reaction the emitted particles have in the average half the beam energy and a very large width in momentum space. These particles predominantly suffered one scattering. With increasing time, the average momentum as well as the width decrease, but only at a very late stage the average momentum coincides with the nucleus nucleus center of mass momentum as expected for particle emission from a compound nucleus. At that stage the system has only little excltation energy left. From this observation we can conclude that the single particle spectra - although exponential in shape - are actually a quite complicated superposition of distributions of particles emitted at different times. This is not surprising: Projectile and target nucleus initially have a Fermi distribution. After one collision between a projectile and a target nucleon we expect for an isotropic cross section a center of mass velocity of the collision partner of half the beam momentum and the momentum distribution being the convolution of the Fermi distribution of projectile and target 
which comes close to a Gaussian. Particles emitted later have more collisions in the average. Therefore their width is reduced and the average momentum has decreased. Only at the very end we see the formation of a compound nucleus, however with small excitation energy. Only very few of the observed particles come from this final stage of the reaction.

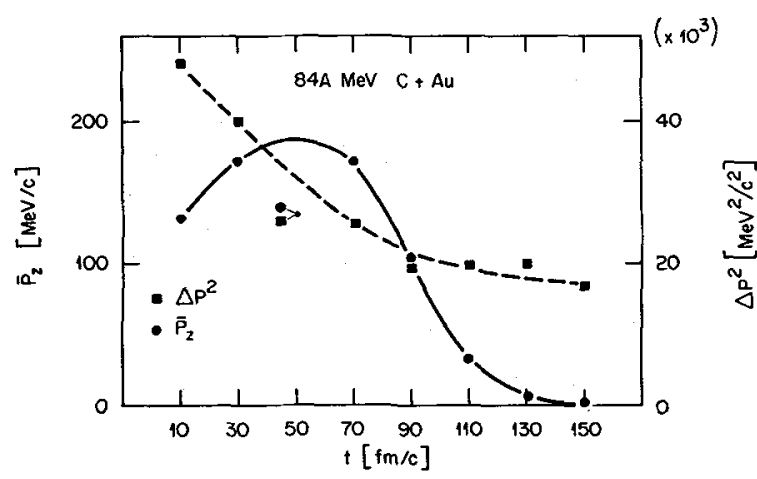

Fig. $7_{2}$ : Mean momentum $\bar{p}_{2}$ and $\Delta p^{2}=\Sigma\left(p_{i}-\bar{p}_{z}\right)^{2} / N$ of the particles emitted in the reaction $84 \mathrm{MeV} / \mathrm{N} \mathrm{C}+\mathrm{Au}$ as a function of the emission time.

Summarizing these observations we see that at intermediate energies the observed particles - although their spectrum is exponential in shape do not come from an equilibrated source as at high and at low beam energies. Almost all of them are emitted prior to equilibrium, having average velocities between nucleon nucleon and nucleus nucleus center of mass velocities, although the width decreases because the excitation energy left in the remnant decreases. So neither is the mean field strong enough to bind projectile and target nucleons long enough for the formation of a compound nucleus nor is the mean free path sufficiently short that equilibration is reached while the projectile travels through the target. The latter is a consequence of the Pauli blocking.

\section{VI - LINEAR MOMENTUM TRANSFER}

one of the primary challenges in intermediate energy heavy ion physics is to determine the linear momentum which can be transferred between nuclei. This quantity determines the excitation energy which can be stored in a nucleus and hence the maximal temperature assuming that a global equilibrium can be obtained. Recently models were advanced $[12,13]$ which rely on very large momentum transfer. We have shown [8] that linear momentum transfer predicted in the BUU approach agrees reasonably with the value measured by Galin et al. [5] for the case 60 $\mathrm{MeV} / \mathrm{N} \mathrm{C}+\mathrm{Au}$. Here we report on an extension of this calculation to determine the target mass and projectile energy dependence of this quantity for central collisions. Figure 8 shows our prediction for four projectile target combinations at three different energies : $60 \mathrm{MeV} / \mathrm{N}$, $84 \mathrm{MeV} / \mathrm{N}, 250 \mathrm{MeV} / \mathrm{N} \mathrm{C+Ni}, \mathrm{C}+\mathrm{Sm}, \mathrm{Ne}+\mathrm{Ni}, \mathrm{Ne}+\mathrm{Sm}$. We see in all cases the momentum transfer being incomplete. Hence the incomplete momentum transfer in heavy ion reactions is not a geometrical effect resulting from the partial overlap of projectile and target. At these energies the momentum transfer is proportional to $A * * 1 / 3$ indicating that twobody collisions are the dominant mechanism for transferring momentum. At all three energies the total momentum transferred is proportional to the projectile mass, indicating that each projectile nucleon transfers 
on the average a momentum which is independent of the size of the projectile.

\section{LINEAR MOMENTUM TRANSFER}

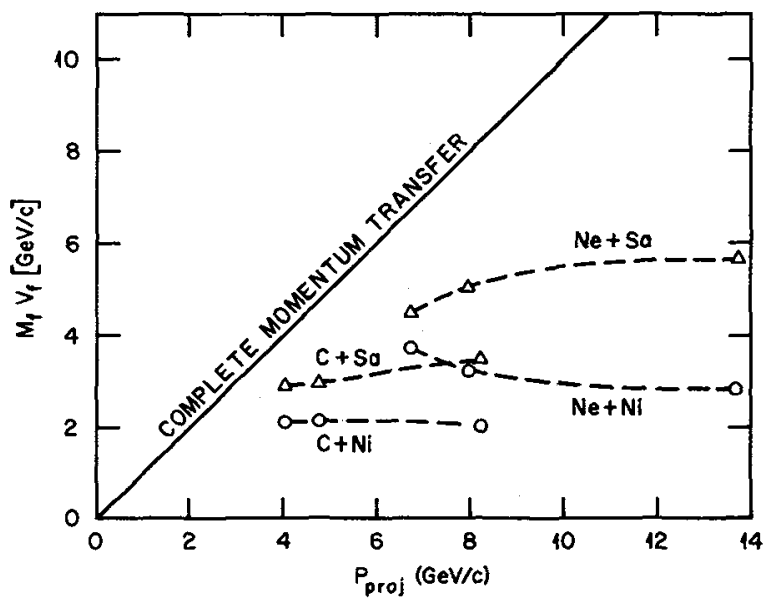

Fig. 8 : Linear momentum transfer as a function of the projectile energy for different systems : Ne+Ni, Ne+Sm, $C+N i, C+S m$.

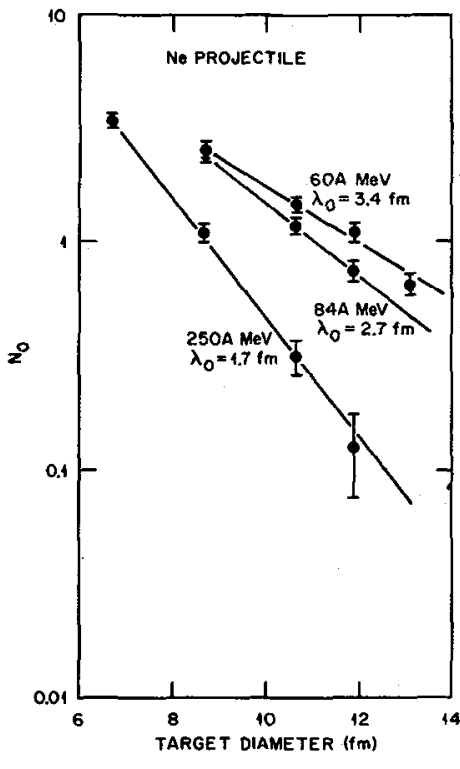

Investigating the momentum transfer as a function of the projectile energy we find that there is a maximal momentum which can be transferred to the system. A further increase of the projectile energy changes this quantity only slightly; for small targets we find even a decrease. This finding agrees with the observations of Galin et al. [5], however, our absolute values are somewhat higher. At $84 \mathrm{MeV} / \mathrm{N}$ we find for central collisions a momentum transfer of $2.2(3.0) \mathrm{GeV}$ for $\mathrm{C}+\mathrm{Ni}(\mathrm{Sm})$ and $3.3(5.1)$ for $\mathrm{Ne}+\mathrm{Ni}(\mathrm{Sm})$. Hence already at this energy the momentum transfer is quite incomplete. The increase of the momentum transfer with energy up to energies around $200 \mathrm{MeV} / \mathrm{N}$ is correlated with a decrease of the mean free path. This can be seen from figure 9. Here the mean free path of the projectile nucleons is calculated for three different beam energies by counting the number of projectile nucleons (N) which have passed the target without a collision at central impact parameters. We see that at $60 \mathrm{MeV} / \mathrm{N}(84 \mathrm{MeV} / \mathrm{N})$ the Pauli blocking is still quite important. It enlarges the expected mean free path for unblocked collision $\lambda=J(I / \sigma / \rho)$ by a factor of $2.0(1.6)$. At $250 \mathrm{MeV} / \mathrm{N}$ the Pauli blocking is unimportant ${ }^{4}$ concerning the mean free path, i.e. the probability to have at least one collision.

Fig. 9 : Number of unscattered projectile nucleons as a function of the diameter of the target for three different beam energies. $\lambda$ denotes the extracted mean free path. 


\section{SUMMARY}

Heavy ion reactions at beam energies between $10 \mathrm{MeV} / \mathrm{N}$ and $200 \mathrm{MeV} / \mathrm{N}$ show a variety of phenomena which is neither known from high energy nor from low energy physics. This is due to the equal importance of the effect of the nuclear mean field and that of two-body collisions. In this transition region we still see signs of the familiar low energy behaviour like formation of a compound nucleus in the late stage of the reaction but also the first hints of typical high energy phenomena like a midrapidity sources. In addition to that new phenomena - like the formation of three cluster - appear which are specific to this energy interval.

Hence one should not wonder that the simple models, which describe high or low energy physics so well, do not work at intermediate energies. We see that the assumptions of a thermalized source of particle emission cannot be verified in contradistinction to reactions at lower or higher beam energies. Neither is the mean free path sufficiently short to thermalize projectile and target matter while traveliing through each other nor is the mean field strong enough to force the nucleons together until complete thermalization is reached. But exactly this is the reason why intermediate energies are so interesting. They offer the unique possibility to study how a many-body system comes to equilibrium which is sufficient in number to have interesting features but contains little enough particles that it can be studied theoretically on a microscopic level.

\section{REFERENCES}

[1] B. Jakobsson et al., Proceedings of the 4th Bergen Workshop in Nucl. Physics (1982), A. Oskarsson, Lund University Report 8303.

[2] H. Rabe, Thesis, Univ. Heidelberg (1986).

[3] D.J. Fields W.G. Lynch, C.B. Chitwood, C.K. Gelbke, M.B. Tsang, H. Utsunomiya and J. Aichelin, Phys. Rev. C30 (1984) 1912 .

[4] G. Bizard et al. Proceedings of the XXIII International winter Meeting on Nuclear Physics, Bormio, 1985.

[5] J. Galin et al., Phys. Rev. Lett. 48 (1982) 1787;

S. Leray, Proceedings, this Conference.

[6] L. Westerberg, D.G. Sarantites, D.C. Hensley, R.A. Dayras, M.L. Halbert and J.H. Barker, Phys. Rev. C18 (1978) 796.

[7] J.P. Bondorf, J.N. De, G. Fai, A.O.T. Karvinen, B: Jakobsson, and J. Randrup, Nucl. Phys. 1333 (1980) 285.

[8] J. Aichelin and G. Bertsch, Phys. Rev. C31 (1985) 1730;

J. Aichelin, Phys. Rev. C33 (1986) $537 ;$

Proceedings of "Phase space approach to nuclear dynamics", Trieste 1985, World Scientific Publishing Co., Singapore.

[9] H. Kruse, B.V. Jacak J.J. Molitoris, G.D. Westfall and H. Stöcker Phys. Rev. C31 (1985) 1770 .

[10] J. Aichel in and H. Stöcker, Phys. Lett. 163B (1985) 59.

[11] M.B. Tsang, Proceedings this Conference.

[12] D. Vasak et al., Phys. Lett. 93B (1980) 243; Nucl. Phys. A428 (1984) $291 \mathrm{c}$.

[13] A.D. Panagiotou et al., Phys. Rev. C31 (1985) 55. 